\title{
Plasma fibronectin concentrations in breast fed and formula fed neonates
}

\author{
H E FRISS, ${ }^{*}$ L G RUBIN, ${ }^{*}$ S CARSONS, $\dagger$ J BARANOWSKI, ${ }^{*}$ AND P J LIPSITZ ${ }^{*}$ \\ ${ }^{*}$ Department of Pediatrics, Schneider Children's Hospital, †Department of Medicine, Long Island Jewish \\ Medical Center, and * + Health Sciences Center, State Univerisity of New York at Stony Brook, New York
}

SUMMARY Plasma fibronectin concentration was measured in neonates of 2 to 5 days of age. Although breast fed and formula fed infants were similar in demographic characteristics, the mean (SD) plasma concentration of fibronectin in 26 breast fed infants, 237 (117) mg/l, was significantly higher than in 27 formula fed infants $(171(91) \mathrm{mg} / \mathrm{l})$. Fibronectin was detected in five colostrum specimens (mean concentration $13.4 \mathrm{mg} / \mathrm{l}$ ). Similar bands were detected after gel electrophoresis of purified adult plasma fibronectin and whole plasma from breast fed and formula fed neonates after staining or immunoblotting. Fibronectin isolated from breast mili also appeared similar to purified plasma fibronectin. It is possible, although unlikely, that fibronectin is absorbed intact from ingested colostrum. Alternatively, a factor(s) might be present in colostrum that contributes to the regulation of plasma fibronectin concentration.

Fibronectin is a high molecular weight glycoprotein found in plasma and on cell surfaces. Plasma fibronectin acts as a non-specific opsonin, augmenting macrophage and neutrophil phagocytosis, and modulating reticuloendothelial clearance of bacteria and intravascular debris. Therefore it has a potentially important role in host defence against infection. The major sources of plasma fibronectin include hepatocytes, macrophages, and endothelial cells. ${ }^{1}$ The concentration of plasma fibronectin is lower in newborn infants compared with children and adults and further decreased in neonates with sepsis. $^{2}$

Human milk has been shown to contain fibronectin that is immunologically cross reactive with plasma fibronectin. ${ }^{3}$ We have compared plasma fibronectin concentrations in healthy, term, breast fed and formula fed neonates. In addition, fibronectin concentrations were measured in colostrum and several commercial infants formulas.

\section{Methods}

\section{STUDY POPULATION}

The study group consisted of healthy term infants who were receiving no medication. Blood for assay was collected two to five days after birth at the time of sampling for state metabolic screening. The following clinical data were recorded: sex, race, method of delivery, indication for caesarean section, birth weight, Apgar scores, and age and weight at time of blood sampling.

Colostrum specimens were obtained from five women, two to six days postpartum. Plasma was obtained from healthy, young adults receiving no medications.

Parental consent for blood sampling was obtained according to guidelines established by the Human Subjects Review Committee of Long Island Jewish Medical Center.

\section{SPECIMEN COLLECTION}

Blood for assay was collected after heelstick puncture into citrate coated capillary tubes (filled with $19 \%$ sodium citrate and baked in an oven until dry). Blood from adults was obtained by venepuncture and injected into citrated tubes (Vacutainer, Becton-Dickinson). Plasma was separated by centrifugation and kept frozen at $-20^{\circ} \mathrm{C}$ until assayed.

Colostrum and mature breast milk specimens were collected into polystyrene tubes after manual expression. Specimens were centrifuged in an Eppendorf microcentrifuge. The fat layer and pellet were discarded and the supernatant was frozen at $-20^{\circ} \mathrm{C}$ until assayed.

Pasteurised whole cows' milk and four commercial infant formulas (Similac, PM 60/40, Isomil (Ross Laboratories), and Prosobee (Mead-Johnson)) were similarly processed. 
FIBRONECTIN ASSAY

Fibronectin concentrations were determined by a modification of the competitive enzyme immunoassay previously reported. ${ }^{4}$ Microtitre plate wells (Dynatech Laboratories) were coated with purified human plasma fibronectin (Cappel Laboratories) $(1 \mathrm{mg} / \mathrm{l}$ in $0.01 \mathrm{M}$ TRIS buffer containing $0.15 \mathrm{M} \mathrm{NaCl}, 0 \cdot 02 \%$ sodium azide, $\mathrm{pH} 7 \cdot 4)$ by overnight incubation at $4^{\circ} \mathrm{C}$. The plates were washed five times with phosphate buffered saline $0 \cdot 05 \%$ Tween 20 and blocked with phosphate buffered saline containing $1 \%$ bovine serum albumin. A 1:20000 dilution of rabbit antihuman fibronectin (Cappel) was incubated with increasing concentrations of fibronectin standard or varying dilutions of plasma, colostrum, or commercial milk preparations overnight at $4^{\circ} \mathrm{C}$ in polystyrene tubes. Antigen antibody mixtures were then transferred to the fibronectin coated, blocked, washed microtitre plate wells and incubated for one hour at $37^{\circ} \mathrm{C}$. Plates were then washed and goat antirabbit IgG conjugated to alkaline phosphatase (Cappel) was added to each well and incubated for one hour at $37^{\circ} \mathrm{C}$. After washing, paranitrophenyl phosphate (Sigma Chemical Company) was added (buffer $0.05 \mathrm{M} \mathrm{NaHCO}_{3}$, $1 \mathrm{mM} \mathrm{MgCl} 2 \mathrm{pH} 9 \cdot 8)$. The plates were observed for colour development and the optical density of the wells was read on a Dynatech MR 600 plate reader at $410 \mathrm{~nm}$. The concentration of fibronectin in each sample was determined by comparing the optical density of test wells with wells containing known concentrations of fibronectin. All samples were run in triplicate and the mean values determined. The coefficient of variation for individual samples was less than $2 \%$. The results of a given assay were discarded if the correlation coefficient of the fibronectin standards and adjusted optical density values on the plate was less than 0.95 .

ISOLATION OF PLASMA AND BREAST MILK FIBRONECTIN Fibronectin was isolated from adult plasma using gelatin-Sepharose chromatography as previously described. ${ }^{4}$ Mature breast milk samples from a single donor were centrifuged at $10000 \times \mathrm{g}$ for 30 minutes, the cream was removed, and the sample was respun at $90000 \times \mathrm{g}$ for 30 minutes. ${ }^{3}$ Fibronectin was isolated from the supernatant sample by gelatin Sepharose chromatography as for plasma fibronectin.

\section{ELECTROPHORESIS AND WESTERN BLOTTING}

Sodium dodecylsulphate polyacrylamide gel electrophoresis (SDS-PAGE) was performed in vertical gels with an acrylamide concentration of $6 \%$ using a Laemmli buffer system. ${ }^{5}$ Samples were reduced with dithiothreitol. Molecular weight standards were purchased from Bio-Rad (Richmond). Gels were stained with Coomassie brilliant blue. Identification of fibronectin species in plasma and milk was accomplished by SDS-PAGE followed by electrophorectic transfer of protein to nitrocellulose sheets. Immunoblotting was completed by sequen-

Table Characteristics of patients in study population

\begin{tabular}{|c|c|c|}
\hline & $\begin{array}{l}\text { Breast fed } \\
\text { neonates } \\
(n=26)\end{array}$ & $\begin{array}{l}\text { Formula fed } \\
\text { neonates } \\
(n=27)\end{array}$ \\
\hline Sex (male/female) & $12 / 14$ & $12 / 15$ \\
\hline Race (white/non-white) & $23 / 3$ & $23 / 4$ \\
\hline $\begin{array}{l}\text { Method of delivery } \\
\text { (\% vaginal delivery) }\end{array}$ & $58^{*}$ & $78^{*}$ \\
\hline Mean (SD) birth weight (g) & $3446(513)$ & $3441(450)$ \\
\hline $\begin{array}{l}\text { Mean (SD) Apgar score at } \\
5 \text { minutes }\end{array}$ & $9 \cdot 7(0 \cdot 46)$ & $9 \cdot 8(0 \cdot 46)$ \\
\hline $\begin{array}{l}\text { Mean (SD) age at time of } \\
\text { sampling (hours) }\end{array}$ & $77(29) \dagger$ & $63(23) \dagger$ \\
\hline $\begin{array}{l}\text { Mean (SD) weight on day } \\
\text { of sampling (g) } \\
\text { Mean (SD) \% birth weight }\end{array}$ & $3301(468)$ & $3373(440)$ \\
\hline lost & $4 \cdot 0(2 \cdot 5) \ddagger$ & $1.9(1.5) \ddagger$ \\
\hline
\end{tabular}

${ }^{*} \mathrm{p}>0 \cdot 1 ; \chi^{2}, 4$ fold table; $\dagger \mathrm{p}>0.05 ; \ddagger \mathrm{p}<0.001$.

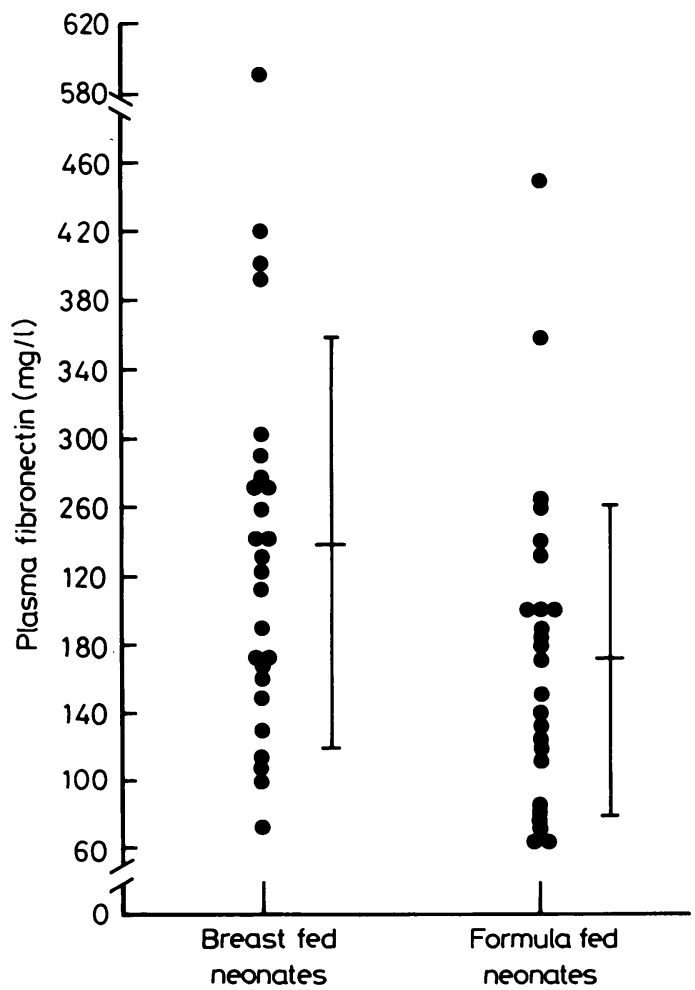

Fig 1 Fibronectin concentrations in breast fed and formula fed neonates. Bars indicate mean and one standard deviation. 
tial incubation of nitrocellulose sheets with bovine serum albumin, rabbit antihuman fibronectin (Cappel), perioxidase conjugated goat antirabbit IgG (Bio-Rad), and perioxidase substrate as previously described. ${ }^{6}$

Data were analysed using the Wilcoxon's rank sum test or $\chi^{2}$ test.

\section{Results}

Of the 26 breast fed and 27 formula fed neonates, there were no significant differences between the two groups in sex, race, method of delivery, indication for caesarean section, birth weight, Apgar scores, or age at time of sampling. The percentage of weight lost from birth to time of sampling was significantly greater in the breast fed group (table); however, the magnitude was only $4.0 \%$ in the breast fed group compared with $1.9 \%$ in the formula fed group.

Plasma fibronectin concentrations were significantly higher in the breast fed newborns com-

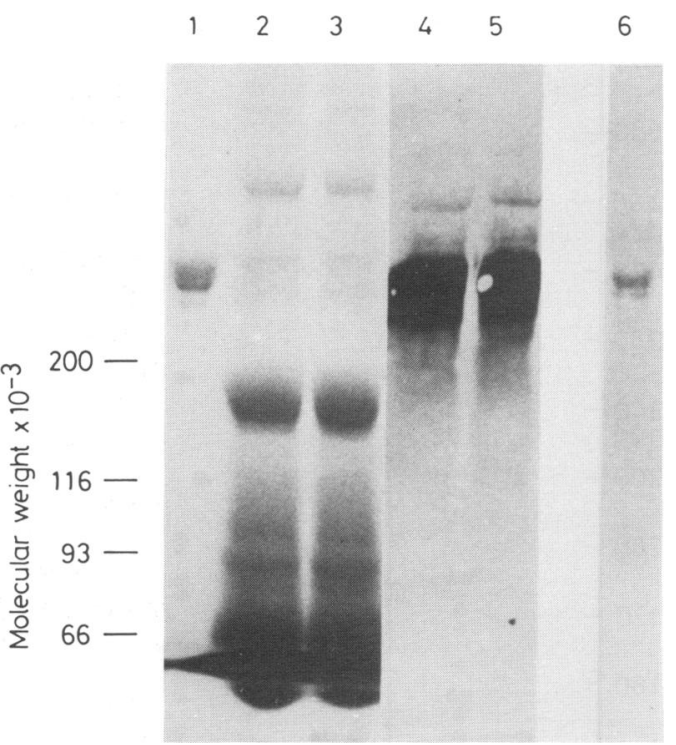

Fig 2 Sodium dodecylsulphate polyacrylamide gel electrophoresis of plasma from breast fed or formula fed neonates and of fibronectin isolated from breast milk. Lanes 1,2 , and 3 were stained with Coomassie brilliant blue. Lanes 4, 5, and 6 were transferred to nitrocellulose and fibronectin was detected using monospecific antibody. Lane 1 contains purified adult plasma fibronectin. Lanes 2 and 4 contain $30 \mu \mathrm{l}$ of pooled plasma from 12 breast fed neonates. Lanes 3 and 5 contain $30 \mu$ of pooled plasma from 12 formula fed newborns. Lane 6 contains fibronectin isolated from human breast milk. pared with the formula fed newborns $(\mathrm{p}<0 \cdot 003$, Wilcoxon's rank sum test). The mean (SD) plasma fibronectin concentration was 237 (117) $\mathrm{mg} / \mathrm{l}$ in breast fed newborns and $171(91) \mathrm{mg} / \mathrm{l}$ in formula fed newborns (fig 1). The mean (SD) plasma fibronectin concentration in 23 healthy adult volunteers was $576.0(252) \mathrm{mg} / \mathrm{l}$ within the range of previously reported values. ${ }^{7}$

The mean (SD) fibronectin concentration in five colostrum specimens was $13.4(4.7) \mathrm{mg} / \mathrm{l}$ (range 6.9-19.5). Fibronectin was not detected in whole cows' milk or four commercial infant formulas. The limit of detection for the assay was $0.05 \mathrm{mg} / \mathrm{l}$.

Coomassie blue staining after SDS-PAGE showed a doublet in the lanes containing purified adult plasma fibronectin and pooled plasma samples from breast or formula fed neonates which corresponded to a molecular weight of roughly 220 kilodaltons (fig 2, lanes 1-3). Electrophoretic transfer and immunoblotting with antifibronectin antibody showed similar bands in lanes containing purified fibronectin, or plasma from breast or formula fed infants (fig 2, lanes 4 and 5). Even with deliberate overloading of lanes with plasma, there were no lower molecular weight immunoreactive fibronectin fragments detected in the plasma of breast or formula fed neonates. Fibronectin isolated from human breast milk also appeared similar to purified human plasma fibronectin (fig 2, lane 6).

\section{Discussion}

Our results support previous studies that have observed lower plasma fibronectin concentrations in neonates in comparison with older infants and adults. $^{7}$ In this investigation we have shown that breast fed neonates have higher plasma fibronectin concentrations than those who were formula fed. The two groups of infants were comparable in demographic and clinical variables. The greater depletion of intravascular volume in the nursing neonates, as evidenced by a greater percentage of weight loss from birth, could be invoked to explain differences in the concentration of a plasma protein; however, even if the entire difference was due to intravascular water, this apparent $2 \%$ difference in weight loss could not explain the $39 \%$ increase in the plasma fibronectin concentration observed. Inadequate nutrition results in a lowering of plasma fibronectin concentrations. ${ }^{89}$ Therefore the lower caloric intake of breast fed neonates in the first days of life compared with those formula fed would be predicted to result in a lower concentration of fibronectin in the plasma of the breast fed neonates, not the raised concentrations observed.

Although there was no significant difference in 
postnatal age between the two groups, the breast fed infants were somewhat older than the formula fed infants. We considered the possibility that the differences in fibronectin concentrations found may in part be influenced by this small difference in age. A significant correlation was found $(\mathrm{r}=0.477$, $\mathrm{p}<0.01)$ between postnatal age and plasma fibronectin concentrations for the entire group of infants. Thus it is possible that the slightly older postnatal age of the nursing infants influenced our findings. ${ }^{10}$ When the two groups were analysed independently, however, the correlation reached significance in the breast fed infants $(r=0.53, p<0.01)$ but not the formula fed infants $(r=0 \cdot 27, p>0 \cdot 10)$. If fibronectin concentrations were influenced solely by age, we would expect to observe a positive correlation between postnatal age and plasma fibronectin concentration in the formula fed group.

Because fibronectin may have an important role in opsonisation and clearance of bacteria, decreased concentrations may be a factor contributing to the neonate's increased susceptibility to and mortality from bacterial infection. Though the difference in plasma concentrations observed in breast fed and formula fed neonates is significant, it is unclear whether the $39 \%$ higher mean concentration in the breast fed infants is important clinically. As the plasma fibronectin concentrations of adults are more than twice that of neonates, it is clear that there are other factors that have a major role in the regulation of plasma fibronectin concentration. It is not known whether this difference in fibronectin concentration is carried beyond the first few days of life.

We hypothesise several possible mechanisms to account for the differences observed. We have confirmed a previous report that documented the presence of fibronectin in colostrum ${ }^{3}$; therefore, direct absorption of fibronectin may be possible. The significant correlation between postnatal age and fibronectin concentrations in the breast fed infants lends support to this hypothesis. As infants who were older would have ingested a larger amount of colostrum, the older breast fed infant might be expected to have higher fibronectin concentrations than his younger counterpart if direct absorption occurred. The similar electrophoretic mobility and immunoreactivity of fibronectin isolated from breast milk and adult plasma precludes the detection of breast milk fibronectin in the plasma of breast fed infants using this method. It is not clear, however, whether a large, protease susceptible protein such as fibronectin could be absorbed intact. Studies have shown that the capacity to absorb macromolecules may be more extensive in the immature small intestine than in the highly developed mature intestine. ${ }^{11}$ In addition, it has been suggested that in the newborn period, a greater quantity of protein ingested by intestinal epithelial cells escapes intracellular proteolysis as a result of immature lysozymal function, and therefore more protein becomes available for subsequent transport out of the cell and into the circulation. ${ }^{11}$ As we assayed immunoreactive fibronectin it is possible that we have measured both intact fibronectin and degraded fragments in plasma. The immunoblot analysis of plasma fibronectin from breast fed infants, however, showed no evidence of fibronectin fragments. Furthermore, the difference in plasma fibronectin concentration in breast fed and formula fed infants is not easily accounted for on the basis of fibronectin in breast milk. A newborn ingests more than $500 \mathrm{ml}$ of colostrum in the first three days of life. ${ }^{12}$ Given a fibronectin concentration in colostrum of $13 \mathrm{mg} / \mathrm{l}$, the infant would ingest $6.5 \mathrm{mg}$ of fibronectin. Assuming a plasma volume of $150 \mathrm{ml}$, even complete absorption of ingested fibronectin from the gastrointestinal tract, without intravascular clearance, could account for only two thirds of the increase in plasma fibronectin concentrations observed in the breast fed infants.

A second hypothesis to explain the difference found is that there may be a factor(s) in breast milk that promotes an increase in fibronectin synthesis. This increased production may be specific for fibronectin or may be part of a generalised increase in protein synthesis by hepatocytes, endothelial cells, or other fibronectin producing cells.

Alternatively, there may be factors unrelated to absorption of fibronectin from the gastrointestinal tract or to fibronectin production. For example, the plasma half life of fibronectin may be prolonged in breast fed infants.

Human milk has been shown to contain several immunologically active elements. Cellular components include macrophages, lymphocytes, and neutrophils; humoral elements include immunoglobulins, lysozyme, and lactoferrin. ${ }^{13}$ Several studies have shown that breast fed infants have lower infection rates than formula fed infants. ${ }^{14} \mathrm{~A}$ significant association has been found between breast feeding and decreased rates of gastrointestinal illness. There is less conclusive evidence that breast feeding may protect against respiratory illness and otitis media. ${ }^{13}$ In addition, there appears to be a protective influence of breast feeding on the risk of developing invasive Haemophilus influenzae type b disease. ${ }^{15}$ It is possible that the raised plasma fibronectin concentrations in nursing infants may represent a means by which breast feeding may contribute to their lower risk of infection. Further 
studies are needed to determine the mechanism that causes the raised plasma fibronectin concentrations in breast fed infants, compared with those who are formula fed, and to determine if this raised concentration persists in later infancy; there is also a need to assess the potential contribution of this quantitative difference in protection of the newborn against infection.

\section{References}

1 Yoder MC, Douglas SD, Gerdes J, Kline J, Polin RA. Plasma fibronectin in healthy newborn infants: respiratory distress syndrome and perinatal asphyxia. J Pediatr 1983:102:777-80.

2 Gerdes JS. Yoder MC, Douglas SD. Polin RA. Decreased plasma fibronectin in neonatal sepsis. Pediatrics 1983;72:877-81.

${ }^{3}$ Zardi L. Destree A. Baize E. Isliker H. Human milk fibronectin: identification of fibronectin fragments by transfer of milk proteins from polyacrylamide gels to nitrocellulose sheets. FEBS Lett 1982:143:105-8.

+ Carsons S, Lavietes BB. Diamond HS, Kinney SG. The immunoreactivity, ligand and cell binding characteristics of rhcumatoid synovial fluid fibronectin. Arthritis Rheum 1985:28:601-12

s Lacmmli UK. Clcavage of structural proteins during the assembly of the head of bacteriophage T4. Anal Biochem 1970):227:68(-5.

- Towbin H, Stachelin T. Gordon T. Electrophoretic transfer of proteins from polyacrylamide gels to nitrocellulose sheets: procedure and some applications. Proc Natl Acad Sci USA 1979:76:4350-4.

7 Barnard DR, Arthur MM. Fibronectin (cold insoluble globulin) in the neonate. J Pediatr 1983;102:453-5.

* McKone TK. Davis AT, Dean RE. Fibronectin. A new nutritional parameter. Am Surg 1985;51:336-9.

"Scott RL. Sohmer PR, McDonald MG. The effect of starvation and repletion on plasma fibronectin in man. JAMA 1982:248:2025-7.

11' Domula M. Bykowska K. Wegrzynowicz Z. et al. Plasma fibronectin concentrations in healthy and septic infants. Eur $J$ Pediatr 1985;144:49-52.

"Walker WA. Absorption of protein and protein fragments in the developing intestine: role in immunologic/allergic reactions. Pediatrics 1985:75(suppl):167-71.

12 McClelland DBL, McGrath J, Samson RR. Antimicrobial factors in human milk. Studies of concentration and transfer in human milk. Studies of transfer to the infant during the early stages of lactation. Acta Paediatr Scand /Suppl/ 1978:271:3-20.

1.3 Lawrence RA. Breast-feeding: a guide for the medical profession. St Louis: CV Mosby, 1985:118.

it Kovar MG. Serdula MK. Marks JS, Fraser DW. Review of the epidemiologic evidence for an association between infant feeding and infant health. Pediatrics 1984:74(suppl):615-38.

15 Lum MK. Ward JI, Bender TR. Protective influence of breast feeding on the risk of developing invasive $\mathrm{H}$ influenzac type $\mathrm{b}$ discase. Pediatr Res 1982:16:151A.

Correspondence and requests for reprints to Dr LG Rubin. Schneider Children's Hospital of Long Island Jewish Medical Center. 271-16 76th Avenue, New Hyde Park, NY 11042, USA.

Accepted 13 November 1987 